\title{
Design of Low Fuel Trajectory in Interior Realm as a Backup Trajectory for Lunar Exploration
}

\author{
By Yuki SATO ${ }^{1)}$, Piyush Grover ${ }^{2)}$ and Shoji YoshiKAwA ${ }^{1)}$ \\ ${ }^{1)}$ Mitsubishi Electric Corporation, Tokyo, Japan \\ ${ }^{2)}$ Mitsubishi Electric Research Laboratories, Massachusetts, USA
}

(Received June 24th, 2013)

\begin{abstract}
In case of a failure on a Hohmann-type translunar trajectory, a reconfiguration of the trajectory that utilizes the three body dynamics of the interior realm of Earth-moon system is proposed. The stable manifold and unstable manifold of a periodic orbit around L1 point extended toward the Earth side have homoclinic intersections. In the proposed method, after detection of a failure on the nominal trajectory, the trajectory is modified by small maneuvers so that the spacecraft can be kicked back by the moon and transferred to the unstable manifold. Then the spacecraft is returned to the moon side through the intersection with the corresponding stable manifold on the Earth side. The periodic orbit is used as a parking orbit so that the amount of delta-v at the moon orbit insertion can be reduced. Since the required amounts of delta-v at each individual maneuver are small throughout the reconfigured trajectory, it can serve as a solution for a backup trajectory in case of a main engine failure. Additionally, the long transfer time of the low-energy trajectory and good operability conditions in the interior realm provide an opportunity for diagnosis and repair of the failure.
\end{abstract}

Key Words: Lunar Exploration, Failure, Trajectory Design, Three Body Dynamics, Interior Realm

\section{Nomenclature}

$\begin{array}{lll}v & : & \text { velocity } \\ \Delta v & : & \text { velocity increment } \\ x, y & : & \text { position in two dimension } \\ \mathrm{r}_{12} & : & \text { distance between Earth and Moon } \\ \mathrm{r}_{1} & : & \text { distance to spacecraft from the Earth } \\ \mathrm{r}_{2} & : \text { distance to spacecraft from the Moon } \\ \mu & : \text { mass ratio of the Moon } \\ E & : \text { Energy } \\ C J & : \text { Jacobi constant } \\ a & : \text { Semimajor axis } \\ \omega & : \text { Angle of apoapse }\end{array}$

\section{Introduction}

Lunar exploration is yet again attracting attention from all over the world. In the $21^{\text {st }}$ century, it was kicked off by SMART-1 launched by ESA in 2003. Since then, Japan (SELENE in 2007), China (Chang'e in 2007 and 2010), India (Chandrayaan in 2008) and United States (ARTEMIS in 2008, LRO in 2009, GRAIL in 2011) have sent spacecrafts to the moon. Within ISECG, Japan (SELENE-2 and SELENE-3), United States (LADEE), ESA (Lunar Lander), India (Chandrayaan-2), and Russia (Luna-Resurs, Luna-Glob) are planning to launch spacecrafts to the moon in the next decade. ${ }^{1)}$

In a practical mission design, it is desirable to consider a method of trajectory reconfiguration in case of failures like main engine anomaly, loss of fuel, missing of lunar orbit injection etc.. In the development of SELENE, a method of reducing the minimum required $\Delta v$ for the spacecraft to be captured by the moon in case of main engine anomaly was proposed, ${ }^{2)}$ where the nominal two-impulse Hohmann trajectory is reconfigured by adopting three-impulse Hohmann transfer. Also proposed was a method of trajectory design which enables the spacecraft to re-encounter the moon even when the original orbit injection is not performed. ${ }^{3)}$

Spacecraft trajectory design based on the three body dynamics (primary body, secondary body, and spacecraft) is subject to considerable research. In this framework, unique properties of the dynamics can be utilized, such as the resonance between motion of the spacecraft and the secondary body, or the periodic orbits around the Lagrange points, where the spacecraft is in equilibrium in the rotating frame. The unique dynamics of three-body problem can be utilized to design transfer trajectory requiring lower $\Delta v$, compared to a Hohmann transfer two-body problem design, which reduces the mass budget.

GENESIS launched by NASA demonstrated sampling of solar wind at L1 periodic orbit of Sun-Earth System in which the spacecraft reached the orbit along the stable manifold of the orbit, stabilized the orbit during the observation for two years, and returned to the Earth through the unstable manifold of the orbit. ${ }^{4)}$ ARTEMIS, launched also by NASA demonstrated lunar transfer through L1 and L2 periodic orbits of Earth-moon system for the first time in the world. ${ }^{5)}$ The trajectory design based on three body dynamics is already in a practical level.

While having a lower fuel nominal trajectory and equipping more scientific instruments on board is appealing, we explore the use of three body dynamics to develop a lower fuel "backup" trajectory in case of failures on a two body nominal 
trajectory. For example, if it turns out that sufficient amount of fuel to reach the lunar orbit on the original trajectory is unavailable due to a failure, a backup trajectory requiring lower amount of fuel can rescue the mission. Since not only the total $\Delta v$, but also the required amounts of $\Delta v$ at each individual maneuver are small in these backup trajectories, it would help to achieve the goal when the attainable $\Delta v$ by a single maneuver is significantly limited due to an anomaly of the main engine.

The inspiration for this work is HITEN, the first Japanese lunar explorer, which tried to transfer the main spacecraft to the moon as its extended mission after completing its main missions such as daughter satellite lunar orbit insertion, double lunar swingby trajectories and Earth aerobraking phases. A trajectory utilizing three body dynamics of both Sun-Earth system and Earth-moon system was proposed and successfully applied to achieve the mission goals. The spacecraft first went to the "exterior realm" of the Earth-moon system and was injected to the unstable manifold of L2 periodic orbit of Sun-Earth system. Next, it transferred onto the stable manifold of a periodic orbit about the Earth-Moon L2 point. Finally, it was transferred to a moon orbit via the unstable orbit of the same periodic orbit. ${ }^{6)}$ Trajectory reconfiguration considered for $\mathrm{SELENE}^{2}$ is similar to this in that it also utilizes the dynamics of the exterior realm of the Earth-moon system.

In this work, a method of translunar trajectory reconfiguration which utilizes three body dynamics of the "interior realm" of Earth-moon system is proposed. In the reconfigured trajectory utilizing the dynamics of interior realm, required amounts of $\Delta v$ at each individual maneuver are significantly smaller than those of trajectory designed based on two body dynamics, although the total amount of necessary fuel does not change much. Another benefit of using interior realm is a good operability of the satellite where faster communication link and better observation are achieved. Since lower fuel trajectories have longer time of flight, it can enable opportunity for diagnosis and repair of the failure before arriving at the moon.

\section{Three Body Dynamics}

Throughout this paper, we discuss the system within the planer circular restricted three-body problem (PCR3BP). The normalized equations of motion of spacecraft in the presence of the Earth and the moon revolving around each other in a plain can be described in the rotating frame as follows,

$$
\begin{gathered}
\ddot{x}-2 \dot{y}=-\bar{U}_{x} \\
\ddot{y}+2 \dot{x}=-\bar{U}_{y}
\end{gathered}
$$

where

$$
\bar{U}(x, y)=-\frac{1}{2}\left(x^{2}+y^{2}\right)-\frac{1-\mu}{r_{1}}-\frac{\mu}{r_{2}}
$$

The distance is normalized by the distance between Earth and the moon. The revolution period of the moon is $2 \pi$ in these units. The first term of Eq. (3) gives the potential energy due to the centrifugal force. The energy $E$ is constant over time unless $\Delta v$ is assigned. $-2 E$ is called Jacobi constant.

$$
\begin{gathered}
E=\frac{1}{2}\left(\dot{x}^{2}+\dot{y}^{2}\right)+\bar{U} \\
C J=-2 E
\end{gathered}
$$

There exist five equilibrium points called Lagrange points, L1 to L5. We focus on the L1 point located between the Earth and the moon. The L1 point is of saddle $\mathrm{x}$ center type, and there exists a family of periodic orbits around the L1 point for certain energy values. There also exist a set of trajectories winding away from the periodic orbit and a set of trajectories winding onto the periodic orbit, constructing the tube shaped unstable manifolds and stable manifolds, both towards the Earth and the moon side.

Fig. 1 shows the L1 point, L1 periodic orbit and the corresponding stable and unstable manifolds when $C J$ is 3.19. L1 periodic orbit provides a channel to connect the Earth side and the moon side. The size of L1 periodic orbit depends on $C J$. Smaller the $C J$, bigger is the size of the periodic orbit. The $\mathrm{L} 1$ periodic orbit exists when $C J$ is lower than 3.2 ( $C J$ of $\mathrm{L} 1$ point). If $C J$ is greater than 3.2, the energy is so small that the channel is closed. If $C J$ is significantly smaller than 3.0 ( $C J$ of L4 and L5 point), the energy is so big that the periodic orbit cannot exist and unique properties of the three body dynamics disappear.

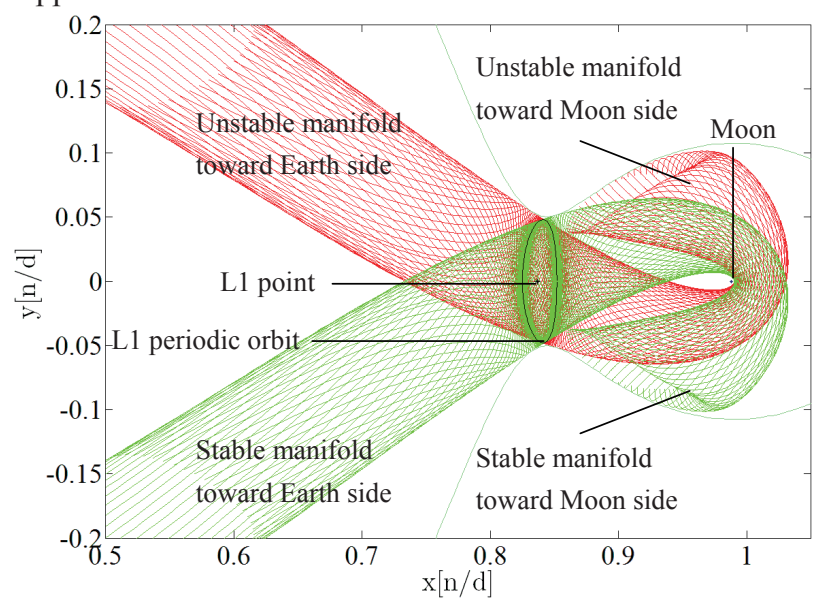

Fig. 1. L1 point, $\mathrm{L} 1$ periodic orbit and manifolds $(C J=3.19)$.

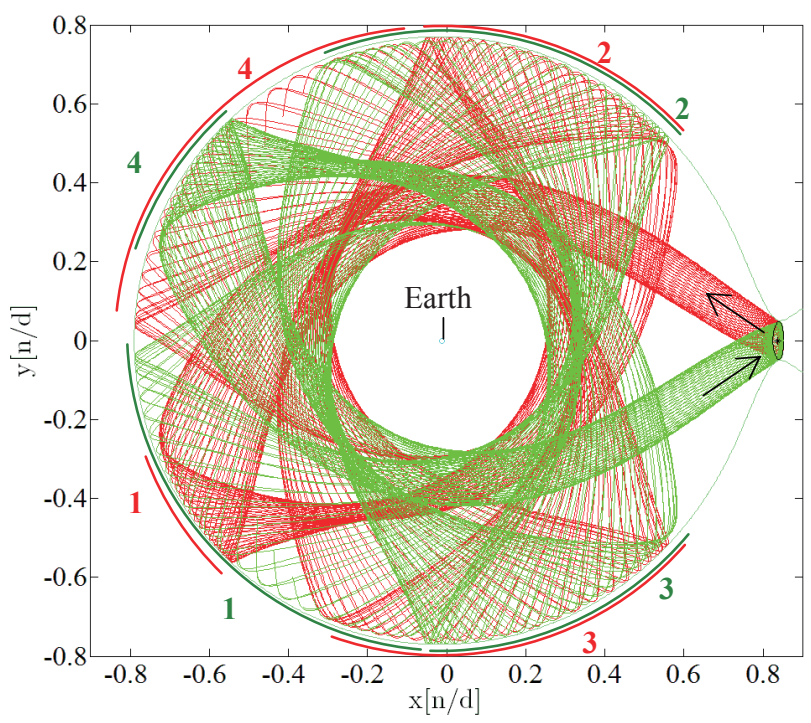

Fig. 2. Stable and unstable manifolds $(C J=3.19)$. 


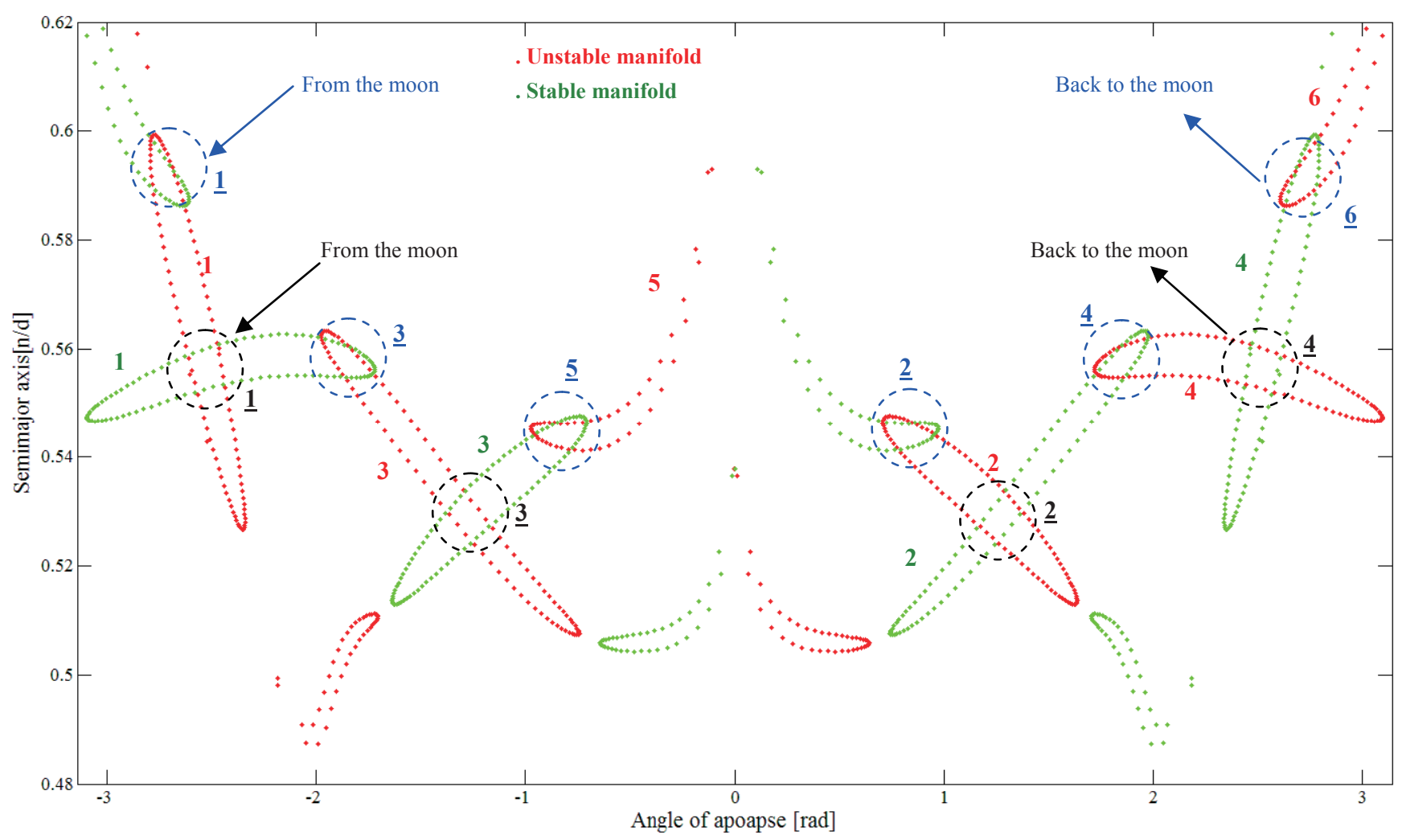

Fig. 3. Homoclinic intersection of the stable and unstable manifolds viewed in Poincare section $(C J=3.19)$.

In Fig. 2 stable and unstable manifolds toward Earth side are numerically extended till they pass apoapse four times. The integers indicate the order of set of the apoaps which each manifold passes as time goes forward. Note that the fourth apoapse of stable manifold in Fig. 2 is the first apoapse in the backward integration since the stable manifold was drawn by giving each position of the periodic orbit a small perturbation in the direction of the eigenvector of the stable eigen value, then integrating backward time.

On the first apoapse section, portions of stable and unstable manifolds overlap. Also the third apoapse of unstable manifold seems to be partly overlapped by it. Fig. 2 shows intersection of these two manifolds projected on configuration space. Poincare sections in Fig. 3 provide a better view of the homoclinic intersection or homoclinic connection. ${ }^{7)}$

Fig. 3 shows a Poincare section of the stable and unstable manifolds projected at the apoapse surface of sections. The integers colored by red and green indicate the order of the set of the apoaps of unstable and stable manifold, corresponding to the integers in Fig. 2. Each state of the manifold at apoapse is converted to two dimensional variables, angle of apoapse $\omega$ and semimajor axis $a^{8)}$ by following way.

$$
a=\frac{\omega=\arctan (y / x)}{-r^{2}+2(\dot{x} y-\dot{y} x)-\left(\dot{x}^{2}+\dot{y}^{2}\right)+\frac{2}{r}}
$$

where

$$
r=\sqrt{x^{2}+y^{2}}
$$

In PCR3BP, the motion of spacecraft is not exactly an ellipse. The semimajor axis in this context is the semimajor axis if the spacecraft trajectory is approximated by ellipse around the apoapse, in other words, semimajor axis of the osculating elliptical orbit. Eq. (7) is derived from the definition of normalized energy in two body dynamics,

$$
2 E=-\frac{1}{a}=\dot{r}^{2}-\frac{2}{r}+\frac{h^{2}}{r^{2}}
$$

where

$$
h=r^{2}+r^{2}\left(\frac{-\dot{x} y}{r^{2}}+\frac{x \dot{y}}{r^{2}}\right)
$$

is the sum of angular momentum due to the rotation of the frame and that of the spacecraft in the rotating frame.

In Fig. 3, the overlaps of projections on first apoapse of the stable manifold with the first and third apoapse of the unstable manifold are the intersections encircled by black dash line with $\underline{1}$ and blue dash line with $\underline{3}$ each other. If the spacecraft is located on the unstable manifold which is going away from the moon side, and if the state of the spacecraft in the unstable manifold is within the intersection with the stable manifold as indicated by black 1 , it will return to the moon side through the stable manifold by revolving around the Earth for four times. The transfer is indicated by the transition of the intersections in the Poincare section encircled by black dash line with $\underline{1}$ to $\underline{4}$. Similarly, if the state of the spacecraft is within the intersection indicated by blue $\underline{1}$, it can again return to the moon by revolving around the Earth for six times. The trajectory is indicated by the transition of the intersections encircled by blue dash line with $\underline{1}$ to $\underline{6}$. 


\section{Trajectory Design Concept}

The main idea of our trajectory reconfiguration plan is to utilize the homoclinic intersection of the unstable manifold and stable manifold of the Earth side to retarget the moon. We assume the original mission plan is to transfer the spacecraft to the moon by a Hohmann-type trajectory. Once a failure is detected on the nominal trajectory and it turns out that originally planned insertion to the moon is impossible, our idea is to give small maneuvers so that the spacecraft is transferred to the unstable manifold of the L1 periodic orbit. Through the homoclinic intersection and the corresponding stable manifold, the spacecraft is again able to return to the moon side.

At least two maneuvers are necessary to transfer the spacecraft from the nominal trajectory to the unstable manifold. The first maneuver is used to deflect the spacecraft away from the nominal trajectory and the second maneuver is used to connect to the trajectory on the unstable manifold. Then spacecraft ballistically revolves around the Earth several times (four or six times according to the way of intersection explained in the previous section) till it reaches the last apoapse of the stable manifold prior to the L1 periodic orbit shown in Fig. 2 and Fig. 3 indicated by integer "4" colored by green. Very small maneuvers can be applied at apses to adjust the state at the last apoapse before arriving. From the last apoapse, we retarget the moon again. The schematic of the trajectory reconfiguration is shown in Fig. 4.

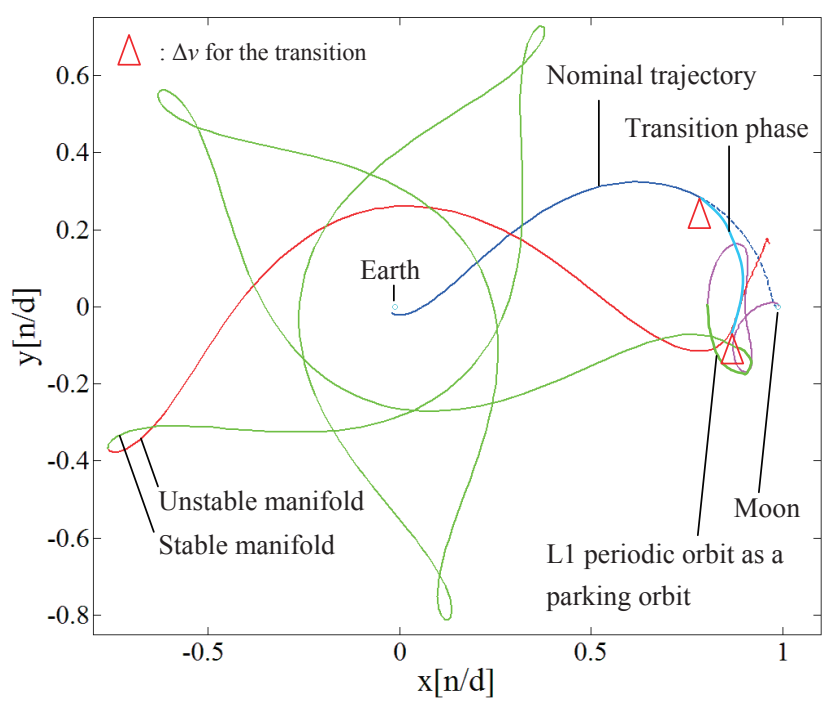

Fig. 4. The schematic of the trajectory reconfiguration.

In the transfer from the last apoapse to the moon, L1 periodic orbit can be utilized as a parking orbit, yielding a significant reduction of the required amount of $\Delta v$ at the moon orbit insertion. This is one of the benefits of using stable manifold as a transfer trajectory. Fig. 5 shows a two impulse Hohmann-type trajectory to the moon in three body dynamics in rotating frame. The initial orbit is Earth parking orbit of perigee height $1000 \mathrm{~km}$ and apogee height $36000 \mathrm{~km}$. The targeted orbit is of periliune height $100 \mathrm{~km}$ and apolune height $11741 \mathrm{~km}$, similar to that of SELENE. As the flight data shows ${ }^{9)}$ the $\Delta v$ at the moon orbit insertion in this case is about
$290 \mathrm{~m} / \mathrm{s}$. On the other hand, Fig. 6 shows a transfer trajectory to the moon from the L1 periodic orbit as a parking orbit. Very small maneuver $(0.3 \mathrm{~m} / \mathrm{s})$ is applied at the periodic orbit to deflect the spacecraft away from it and get the spacecraft on the unstable manifold tube extended toward the moon side. Then the spacecraft is ballistically transferred to the region near to the moon, and the second maneuver is applied to insert it to the target orbit. The $C J$ of the periodic orbit is 3.19. The trajectory takes about 12 days. The $\Delta v$ at the insertion to the same target orbit takes only $88.3 \mathrm{~m} / \mathrm{s}$. This can rescue the mission when the maximum attainable $\Delta v$ by single maneuver is significantly limited due to an anomaly of main engine.

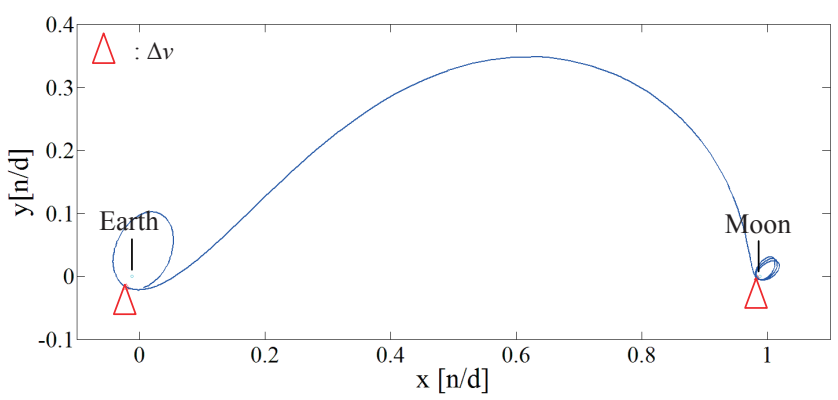

Fig. 5. Hohmann transfer in rotating frame ( $\Delta v$ for insertion is $290 \mathrm{~m} / \mathrm{s}$ ).

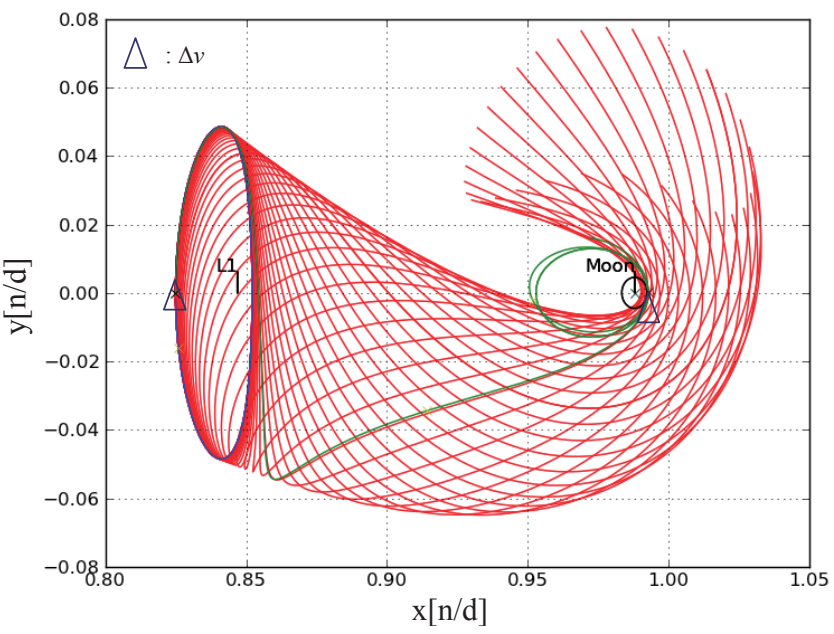

Fig. 6. Transfer trajectory to the moon from the $\mathrm{L} 1$ periodic orbit $(C J=$ $3.19, \Delta v$ for insertion is $88.3 \mathrm{~m} / \mathrm{s}$ ).

In the design of backup trajectory described above, we decompose the whole trajectory into four phases and optimize them respectively in a given order. The phases are transition phase from the nominal trajectory to a trajectory on the unstable manifold, the ballistic revolution phase around the Earth till the spacecraft reaches the last apoapse of the stable manifold, insertion phase from the last apoapse to the L1 periodic orbit, and the insertion phase from the L1 periodic orbit to the moon.

\section{Optimization}

Since we assume that the backup trajectory is used when a failure like main engine anomaly or loss of fuel occurs, the amount of $\Delta v$ at each maneuver and also their total should be smaller than that of the moon insertion maneuver of the nominal trajectory, $290 \mathrm{~m} / \mathrm{s}$. 
The last three phases of the backup trajectory are well researched. In the previous work, we showed that targeting the L1 periodic orbit from inside the tube of stable manifold (third phase) needs only around $2 \mathrm{~m} / \mathrm{s}^{8}{ }^{8}$ Typical example of targeting the moon from the L1 periodic orbit (forth phase) is shown in Fig. 6. Although the required $\Delta v$ depends on $C J$ of the L1 periodic orbit, it is significantly smaller than $290 \mathrm{~m} / \mathrm{s}$.

In this work, we first show the existence of the first phase of the proposed backup trajectory. Based on an iterative search among different orbit topologies, we found that a three-impulse maneuver can provide a transfer from Hohmann-type nominal trajectory to the unstable manifold with acceptable level of $\Delta v$. In particular, the first maneuver is performed on the nominal trajectory to increase the energy and have the spacecraft go to the far side of the moon. Then it is kicked by the moon. The second maneuver is assigned near the moon to adjust the direction of the trajectory. The third maneuver is assigned at the first perigee after the kick to reduce the energy and connect the trajectory to the unstable manifold.

We optimize this trajectory by multiple shooting method given a fixed initial point on the nominal trajectory. The starting point is 3.5 days after leaving the Earth parking orbit, 1.7 days before arrival at the moon in nominal plan.

The trajectory to be optimized starts from the fixed initial point, and ends at the first apogee after the moon kick. The parameters are times of the three maneuvers, amount of $\Delta v$ for three maneuvers in each direction, states where the maneuvers are assigned, and the state of the end point as shown in Eq. (11). The objective function is the sum of squares the $\Delta v$ at the three maneuvers as shown in Eq. (12). In addition to equality constraints for continuity and apse condition shown in Eqs. (13) and (14), inequality constraints are assigned to the range of time, $\Delta v$, and $C J$.

$$
z=\left[t_{1}, X_{1}, \Delta v_{1}, t_{2}, X_{2}, \Delta v_{2}, t_{3}, X_{3}, \Delta v_{3}, t_{4}, X_{4}\right]
$$

where $X_{i}=\left[x_{i}, y_{i}, v_{x i}, v_{y i}\right], \Delta v_{i}=\left[\Delta v_{x i}, \Delta v_{y i}\right]$.

$$
\begin{gathered}
J=\sum_{i=1}^{3}\left(\Delta v_{x i}^{2}+\Delta v_{y i}^{2}\right) \\
f\left(X_{i-1}+\left[0,0, \Delta v_{i-1}\right], t_{i}\right)-X_{i}=0 \quad(i=1 \ldots 4)
\end{gathered}
$$

where $X_{0}$ is state of the initial point, and $\Delta v_{0}=[0,0]$.

$$
x_{i} \cdot v_{x i}+y_{i} \cdot v_{y i}=0 \quad(i=2 \ldots 4)
$$

The most important inequality constraint is $C J$ at the end point. It dominates required amount of $\Delta v$ throughout the trajectory. Since the $C J$ of Hohmann-type nominal trajectory is very low (energy is very high comparing with energy of typical low fuel trajectory), higher the $C J$ at the end point is (lower the energy) larger the require amount of $\Delta v$ is.

Figs. 7 and 8 show the optimized trajectory when the minimum of $C J$ at the end point is constrained to be 2.9. Fig. 9 is a close up view of the trajectory in inertial frame.

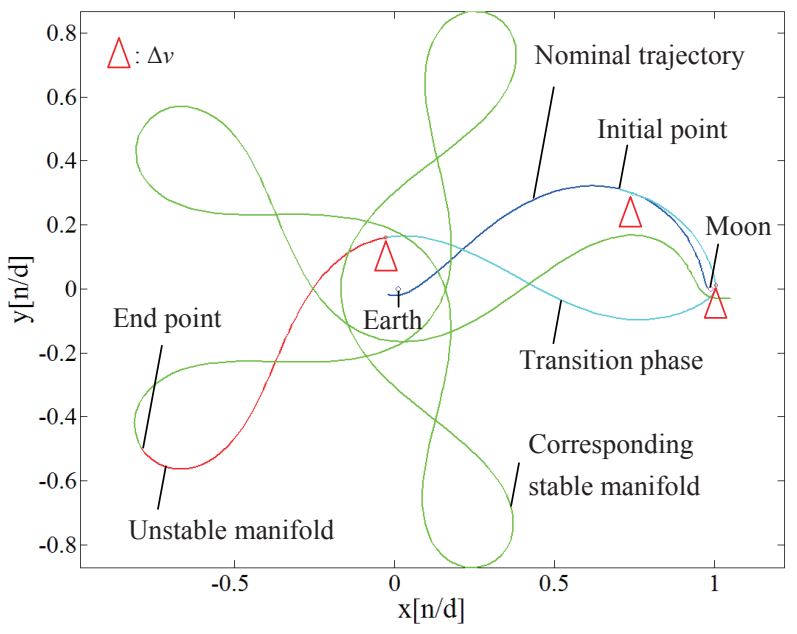

Fig. 7. Optimized first phase of backup trajectory in rotating frame (The minimum $C J$ at the end point is constrained to be 2.9).

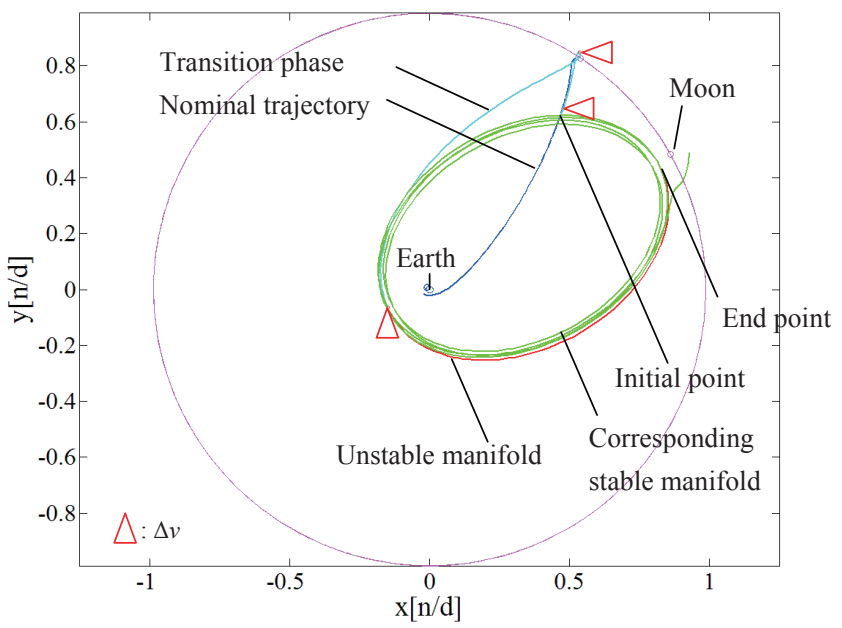

Fig. 8. Optimized first phase of backup trajectory in inertial frame (The minimum $C J$ at the end point is constrained to be 2.9).

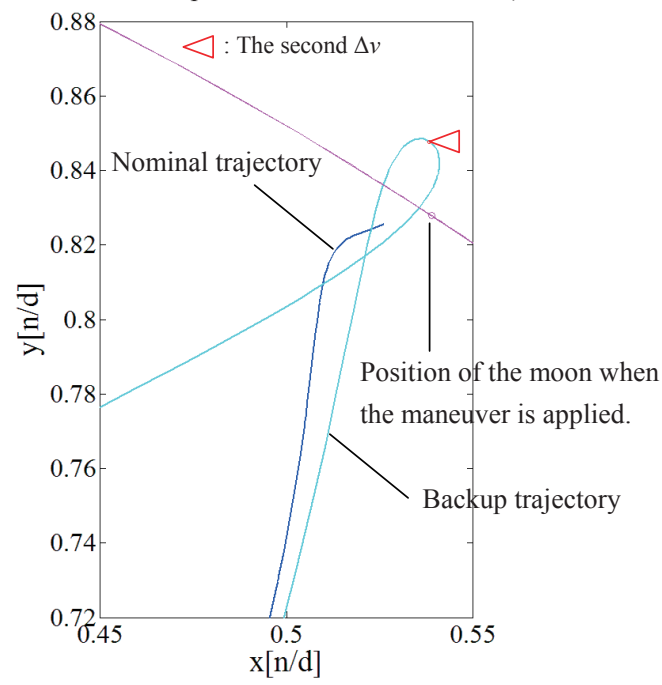

Fig. 9. Close up view of the trajectory in inertial frame.

The corresponding stable manifold is also shown. The first $\Delta v$ is $89.7 \mathrm{~m} / \mathrm{s}$, the second $\Delta v$ is $19.3 \mathrm{~m} / \mathrm{s}$, and the third $\Delta v$ is $87.9 \mathrm{~m} / \mathrm{s}$. These are significantly smaller than $290 \mathrm{~m} / \mathrm{s}$ and might be attainable even when the output of main engine is limited due to a failure. On the other hand, expected amount of total required fuel for whole the backup trajectory is almost 
the same or might be bigger than that of nominal trajectory $(290 \mathrm{~m} / \mathrm{s})$. This is because sum of the three maneuvers (196.9 $\mathrm{m} / \mathrm{s}$ ) and the $\Delta v$ in the forth phase with L1 periodic orbit of $C J$ $3.19(88.3 \mathrm{~m} / \mathrm{s})$ is $285.2 \mathrm{~m} / \mathrm{s}$, and other small maneuvers are necessary for increasing $C J$ from that of the end point to 3.19 in the second phase and for targeting L1 periodic orbit in the third phase. Calculation of the particular small maneuvers and global optimization to reduce the total amount of $\Delta v$ throughout the backup trajectory is currently underway and will be pursued in a future publication.

We optimized the trajectories with different minimum $C J$ values at the end point. Fig. 10 shows the value of $C J$ at initial point (step 0 ), the three points where maneuvers are assigned (step 1, 2, 3), and the end point (step 4) for the different inequality constraints. The $C J$ of step 1 to 3 are calculated for state immediately before the maneuvers are assigned. For all cases, the $C J$ is reduced after the first maneuver so that the spacecraft can go to the far side of the moon. At the third maneuver, $C J$ is increased to its lower bound at the end point so that the required amount of $\Delta v$ at the maneuver can be minimized.

The amount of $\Delta v$ at the three maneuvers for different inequality constraints are shown in Fig. 11. As mentioned above, the smaller the $C J$ at the end point is, smaller the required amount of $\Delta v$. However, the amount of $\Delta v$ for increasing $C J$ to 3.19 in the second phase might be larger. Sum of $\Delta v$ at the three maneuvers is $168.8 \mathrm{~m} / \mathrm{s}(C J \mathrm{~min}=2.8)$, $196.9 \mathrm{~m} / \mathrm{s}(C J \min =2.9), 233.9 \mathrm{~m} / \mathrm{s}(C J \min =3.0), 281.0 \mathrm{~m} / \mathrm{s}$ $(\mathrm{C} J \min =3.1)$.

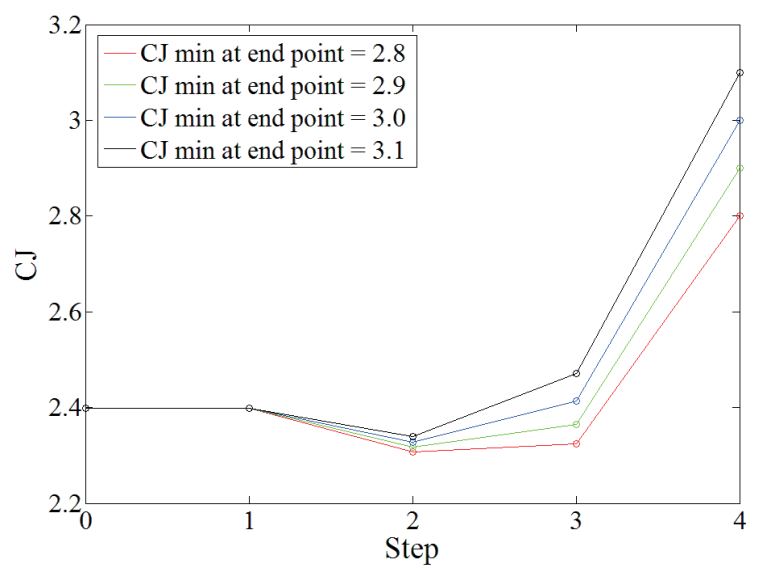

Fig. 10. History of $C J$ different minimum of $C J$ at the end point.

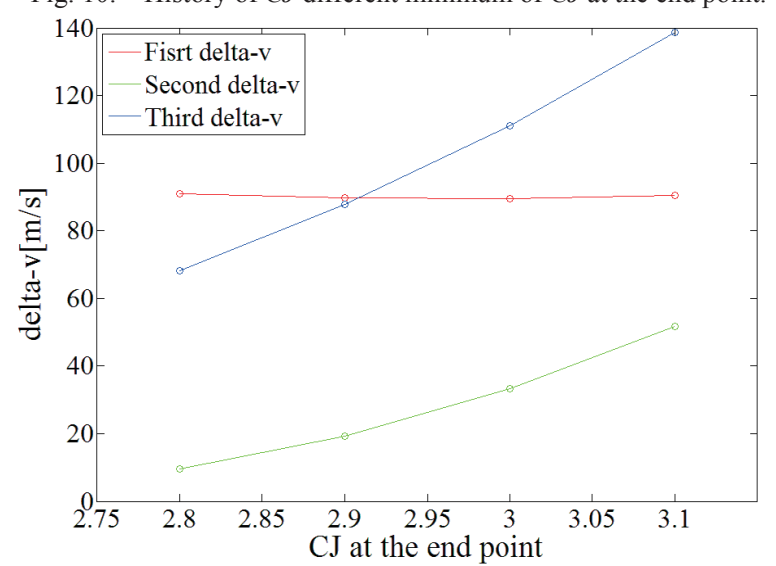

Fig. 11. Amount of $\Delta v$ for different minimum of $C J$ at the end point.

\section{Conclusions}

In case of a failure on a Hohmann-type nominal translunar trajectory, a trajectory reconfiguration that utilizes three body dynamics of the interior realm of the Earth-moon system is proposed. The reconfigured trajectory, called the 'backup trajectory', utilizes the unique dynamics of the stable and unstable manifold of L1 periodic orbit. When a failure is detected, the spacecraft returns to the Earth side, and then reaches an orbit the around moon through the homoclinic interaction of the manifolds.

In this work, we showed the existence of such a backup trajectory with acceptable level of amount of $\Delta v$. Global optimization of the whole trajectory is currently under investigation.

Although the total amount of necessary fuel might be greater than that of nominal trajectory, the low fuel trajectory in interior realm as a backup trajectory provides the following two benefits. The first benefit is the reduction of the required amount of $\Delta v$ at each individual maneuvers. This would offer hope if the attainable $\Delta v$ by single maneuver is significantly limited due to an anomaly of main engine. In particular, the proposed three-impulse maneuver to target the spacecraft to unstable manifold from the nominal trajectory requires 89.7 $\mathrm{m} / \mathrm{s}, 19.3 \mathrm{~m} / \mathrm{s}, 87.9 \mathrm{~m} / \mathrm{s}$, respectively. These are significantly smaller than that of the moon insertion maneuver of the nominal trajectory, $290 \mathrm{~m} / \mathrm{s}$, and might be attainable even when the output of main engine is limited due to a failure.

The second benefit, which is not available in a trajectory reconfiguration utilizing the exterior realms, is good operability of the satellite where faster communication link and better observation are achieved. Since lower fuel trajectory has longer transfer times, it provides an opportunity for diagnosis and repair of the failure before arriving at the moon.

\section{References}

1) ISECG (International Space Exploration Coordination Group): Global Exploration Roadmap, 2011.

2) Kawakatsu, Y:: SELENE Translunar Trajectory Reconfiguration Plan Provided for the case of Main Engine Anomaly, Proceedings of The $20^{\text {th }}$ International Symposium on Space Flight Dynamics, 2007.

3) Kawakatsu, Y.: Study on Lunar Approach Strategy Tolerant of a Lunar Orbit Injection Failure, Trans. JSASS Space Tech. Japan, 5(2007), pp.1-7.

4) Burnett, D.S. et al,: The GENESIS Discovery Mission: Return of Solar Matter to Earth, Space Science Reviews, 105(2003), pp.509-534.

5) Folta, D.C. et al: Application of Multi-body Dynamical Environments: The ARTEMIS Transfer Trajectory Design, Acta Astronautica, 73(2012), pp. 237-249.

6) Belbruno, E.A. and Miller, J.K.: Sun-perturbed Earth-to-Moon transfers with ballistic capture, Journal of Guidance, Control and Dynamics, 5(1993), pp.770-775.

7) Koon, W. et al,: Dynamical Systems, the Three-Body Problem and Space Mission Design, Marsden Books, pp. 60-72, 2008.

8) Grover, P. and Anderson, C: Optimal Three Body Assist and Manifold Transfers in End-to-end Lunar Mission Design, Proceedings of the 22 ${ }^{\text {nd }}$ AAS/AIAA Space Flight Mechanics, 2012.

9) Matsumoto, S. et al: Flight Result of Selenological and Engineering Explorer "KAGUYA" on Lunar Orbit, The $22^{\text {nd }}$ International Symposium on Space Flight Dynamics, 2009. 